\title{
The implications of automobile parts supply network structures: a complex network approach
}

\author{
Tomomi Kito a, b, *, Kanji Ueda (1) c \\ a Department of Systems Innovation, School of Engineering, The University of Tokyo, Tokyo, Japan \\ b Saïd Business School, University of Oxford, Oxford, UK \\ c The University of Tokyo, Tokyo, Japan
}

\begin{abstract}
This study investigates the temporal structural transitions of automobile supply networks where, under situations with various possible risks such as economic crises and market globalisation, companies must both work together for sustainable production, and also compete with each other for their own survival. The resulting network complexity is captured by large-scale empirical data, and analysed for the first time using concepts and metrics developed in complex network science. We discuss how network structure reflects the effect of several factors including market fluctuation, product standardisation, technological advancement, and financial interdependencies between companies.
\end{abstract}

Complexity; Structural analysis; Supply network

\section{Introduction}

Increasing awareness of the complexity in real-life supply networks has lead us to view supply chain management (SCM) from a different perspective; namely that of complex network science. From this perspective, the structure of a given supply network can be captured as the result of emergence, through a multiplicity of repetitive decision-makings and interactions of individual companies pursuing their own profit and survival [1][2]. However, despite the various models and simulations that have been presented (e.g. [3]), the validity of these studies is threatened by unsubstantiated assumptions of how supply networks are formed. The real-world complexity of supply network structures can only be captured by empirical data and the use of appropriate tools to extract relevant information from the data.

This study aims to investigate the structural features of real-life supply networks, by employing a complex network approach. The supply networks under investigation are those of different automobile parts, each network consisting of car assemblers in Japan and their direct suppliers that produce each product. We demonstrate how various factors such as market fluctuation, product standardisation, technological advancement and financial interdependencies between companies may result in the heterogeneity of network structures, and their temporal transitions. Section 2 reviews the existing literature on Japanese automobile supply networks and complex network studies in SCM, through which we clarify the motivation, challenges and novelty of this study. Section 3 explains the empirical data that we collected, and the tools developed in the field of complex network science that are used in this paper. Section 4 presents the results and implications of our analysis, and in section 5, we conclude the paper with a discussion on the significance and limitations of this study, as well as the potential future work.

\section{Research background}

\subsection{Literature review on automobile parts supply networks}

Since the late 1980 's, the distinctive supply network structure of Japanese car assemblers (based on long-term transactional and financial relationships between them and their suppliers: the socalled 'keiretsu'), has been of great interest to researchers across various fields including manufacturing engineering (e.g. [4]). This structure has been cited as enhancing the competitive edge of Japanese car assemblers and their product suppliers in the global marketplace [5], but has also been criticised as creating high entry barriers for independent/foreign companies (although it has been pointed out that Japanese assemblers procure products from multiple suppliers, and Japanese suppliers supply multiple assemblers [6]).

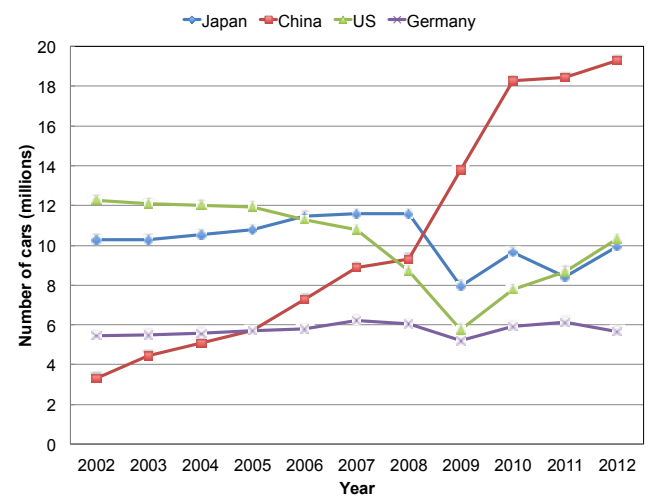

Figure 1. Annual car production in Japan, China, the US and Germany between 2002-2012.

The situation surrounding the Japanese automotive industry, however, has drastically changed since the year 2000. Figure 1 shows the annual car production in Japan, China, US and Germany between 2002-2012. The auto industries in Japan, America and Europe seem to be no longer growing (if not slumping) and appear to have been negatively affected by the rapid growth of the Chinese industry and the 2008 Lehman Crisis. Furthermore, product modularisation and standardisation have resulted in cheaper costs and global procurement strategies, leading to the demise of the aforementioned keiretsu systems [7]. On the other 
hand, the rise of hybrid and electric cars is a driver that is likely to change the industrial structure. It is clear that supply networks of different products will reflect the effects of the aforementioned factors differently, and that companies employing different strategies will form different supply networks. However, to date, the heterogeneity and changes in supply network structures in the $21^{\text {st }}$ century have not been empirically observed. Such analysis requires detailed empirical data on the supply networks, as well as the appropriate set of analytical tools.

\subsection{Complex network approach in SCM}

A complex network approach provides a set of tools that can quantitatively analyse such structural heterogeneity of networks. Complex network science has its origins in discrete mathematics and graph theory, and has been developed over many decades as a theoretical framework for the understanding of the structural characteristics of networks. The interdisciplinary study of complex networks, inspired by real-world networks such as the Internet, genetic regulatory processes, human interactions and so on, has led to the development of many empirical network metrics and models that have been successfully applied across a wide range of research fields.

The complex network perspective has recently been increasingly accepted in the field of management, including SCM. There is compelling evidence that the structure of an organisational network affects the essential measures of its performance, such as the operational efficiencies and its sustainable market competitiveness (e.g. [8][9]), and that the performance of a supply network is also influenced by its structure [10]. A few studies have analysed real-life supply network by applying the complex network metrics [11][12][13].

Although these extant empirical studies provided us with significant insights that could not have been achieved otherwise, the above-mentioned heterogeneity of different product's networks has not yet been investigated due to a lack of relevant data. Therefore, the collection of data on supply networks of different products in itself is novel, and should provide us with new insights. Furthermore, due to the exceptional difficulties of collecting time series data in order to capture the temporal transitions of a supply network of a specific product, to date there has been no studies that have shown such transitions. In this paper, we carry out this challenge with an aim to demonstrate the advantage of obtaining and analysing empirical time-series data.

\section{Empirical data and complex network analysis}

\subsection{Data collection}

As our data source, we used a regularly published series of surveys, each of which providing quantitative information on 200 auto-parts [14]. The information includes the names of suppliers that produce each product, the amount produced, and to which assembler(s) each supplier supplies that product. Since the focus of this paper is only on the structural transitions of supply networks from 2000 onwards, we used who-supplies-what-towhom information from 2002, 2005, 2008, 2010 and 2012. We also researched the company histories to find financial relationships between each company.

\subsection{Complex network analysis methods}

In terms of the complex network approach, a supply network can be represented as a set of nodes and links, where nodes correspond to companies (i.e. assemblers and suppliers), and links correspond to supply relationships, respectively. A given node pair can have multiple types of links; in our case, financial relationships can also be represented as a set of links.
As the first step of our network analysis, we developed a tool that can visualise the supply network structures. Figure 2 shows examples of the visualised structure. The figure on the left captures the supply network of tyre products in 2002, and the one on the right captures the network in 2012. In both figures, the red nodes arranged in the outer circle represent Japanese car assemblers (11 of them in 2002, 12 in 2012), and the blue nodes in the inner circle represent suppliers. The size of the nodes is uniform and hence does not reflect the company's size or capability. A supplier node has a link shown as a grey arrow to an assembler, to whom it supplies the product (i.e. tyre). Note that the thickness of the links is also uniform and hence does not reflect the trade volume. In addition, green arrows are used to show future mergers or acquisitions that occur sometime within the range of the study. In our visualisation, those suppliers who will appear or disappear during the range of the study are represented with different colours (For example, the colour brown means that a company will be acquired at some point); in this example, the brown-coloured supplier in 2002 disappears from the market and does not exist in 2012. This is because it was acquired by the company with which it is linked by a green arrow in the 2002 network. By this means, we can obtain intuitive understandings of the structural features, their temporal changes, and the causality of the changes.

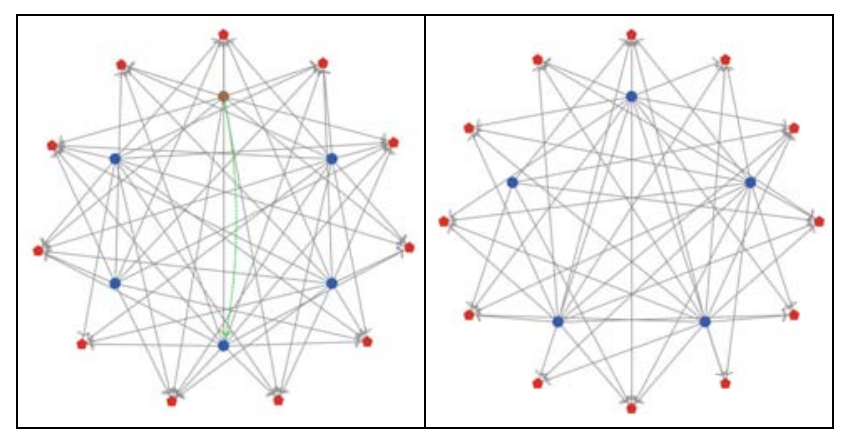

Figure 2. The supply network structures of tyres (left: 2002, right: 2012).

The supply network structures of tyre products (Figure 2) reflect the effects of assemblers' multiple procurement strategy and suppliers' multiple sourcing strategy: each assembler has 2-6 incoming links, and each supplier has 5-12 outgoing links. In the terms of complex network analysis, the number of in-links of a given node is defined as the "in-degree" and the number of outlinks is defined as the "out-degree". These are often used as metrics indicating the centrality of the node, and the degrees of interdependencies amongst nodes. In the case of tyre products, assemblers are not heavily dependent on any specific supplier, and suppliers are also not dependent on any specific assembler. The implication of the degrees of dependency will be discussed in the following section, together with the other products' supply networks.

In this paper, we also applied a metric called 'nestedness', that was developed in the field of ecology. 'Nested' patterns have been observed in various real-life mutualistic networks, which are composed of mutually beneficial interactions between individual nodes of two distinct sets. In a nested network, the links are organised such that 'specialists' (those who have a limited number of links) link with proper subsets of the nodes, whom 'generalists' (those who have many links) link with [15]. In ecology, this nested pattern has been shown to minimise competition between species and therefore provide the network with greater biodiversity and higher sustainability. The more nested a network is, the more sustainable it is. The quantified measure of nestedness [16] has also been successfully applied to 
a supply network [17], proving its usefulness in the field of management.

Since the absolute value of nestedness, $N$, differs depending on the size of a given network, the relative value must be evaluated by comparison to the nestedness value calculated in null models. The null model is a network which matches the network under investigation in some of its structural features, but which is otherwise taken to be an instance of a randomised network. In this paper, we evaluated the significance of the observed nestedness value, by comparison to the average of the calculated nestedness value of 1000 randomised versions of the original network, where links were rewired at random, under the constraint that the expected degree of each node matches the degree of the node in the original network [18]. The relative nestedness value, $N_{r}$, is formalised as follows:

$$
N_{r}=N-N_{\text {ave }} / N_{\text {dev }}
$$

Here, $N_{\text {ave }}$ and $N_{d e v}$ are the average value and standard deviation, respectively, of $N$ calculated for 1000 null models.

\section{Results}

\subsection{General features of the Japanese auto parts supply networks}

Table 1 summarises the general features of the supply networks in the 5 years. The supply network in each year is composed of the Japanese assemblers, suppliers producing any of the 200 products in the specified year, and supply links formed between the assemblers and suppliers. One additional assembler appeared in the data in 2005: Mitsubishi Fuso Truck and Bus, demerged from Mitsubishi Motors (started operation in 2003). Despite the increase in the number of assemblers, the total numbers of suppliers and supply links are found to have been moderately decreasing, reflecting the downturn of the Japanese automotive industry. The consequent monotonic decrease of the average indegree of assemblers implies that the severe market situation has been discouraging the assemblers from using multiple procurement strategies (the average number of suppliers was reduced by $\sim 20 \%$ ), which, as a result has increased the dependency of assemblers on their suppliers. The monotonically increasing tendency of the average out-degree of suppliers (along with the decrease in their total number) implies that the market selection process may have been eliminating those suppliers which were exclusive to specific assemblers, and been reinforcing the existence value of those which supply multiple assemblers.

While these averaged numbers are useful, we found that the number of suppliers in each of the 200 product markets varies from 2 to 30 , indicating a very high degree of heterogeneity between the network structures. This underlines the danger of relying on traditional statistical measures, and the advantages of employing the complex network approach.

\section{Table 1}

General features of the supply networks.

\begin{tabular}{|c|c|c|c|c|c|}
\hline & 2002 & 2005 & 2008 & 2010 & 2012 \\
\hline No. of suppliers & 634 & 611 & 581 & 569 & 561 \\
\hline No. of assemblers & 11 & 12 & 12 & 12 & 12 \\
\hline $\begin{array}{c}\text { No. of links } \\
\text { Average in-degree of } \\
\text { assemblers }\end{array}$ & 2154 & 2193 & 2150 & 2120 & 2112 \\
\hline $\begin{array}{c}\text { Average out-degree of } \\
\text { suppliers }\end{array}$ & 3.40 & 50.9 & 48.4 & 47.4 & 46.8 \\
\hline
\end{tabular}

\subsection{Analysis of supply networks of different products}

Various patterns of supply network structures were observed in our analysis. With the aim of demonstrating how different factors may be reflected in different structural patterns, this paper focuses on, and conducts detailed analysis of, three different types of products.

\section{(a) General products}

A common example of general auto parts would be tyres, of which the supply network structures are shown in Figure 2. The suppliers in this market are not financially affiliated with any assemblers, and supply many assemblers. The average in-degree of assemblers has been slightly decreasing (5.45 in 2002, 4.25 in 2012), which may be due to the acquisition of one supplier by another as explained in section 3.2. Table 2 shows the absolute and relative nestedness values in each year for the tyre network. The high absolute and relative nestedness values indicate the sustainable co-existence of a few suppliers. This could also suggest that it is difficult for small suppliers to enter the market, but at the same time, there is a chance that a more costadvantageous supplier may supersede one of the existing ones.

We found that the markets of other modularised general products such as batteries also show similar trends.

Table 2

Nestedness values of the tyre supply networks.

\begin{tabular}{|l|c|c|c|c|c|}
\hline & 2002 & 2005 & 2008 & 2010 & 2012 \\
\hline$N$ & 41.3 & 43.4 & 46.1 & 44.5 & 48.5 \\
\hline$N_{r}$ & 26.5 & 39.9 & 40.9 & 31.3 & 34.5 \\
\hline
\end{tabular}

\section{(b) In-house manufactured products}

The supply networks of in-house manufactured products (i.e. those which are manufactured by assemblers themselves) were found to show totally different structural features. Engine blocks are one such example: the supply network structures of which are shown in Figure 3. In this figure, the assemblers coloured in pink are also manufacturers of the engine blocks. Red arrows indicate financial relationships. One supplier (coloured in yellow in the 2002 network) disappears by merger with the assembler to which it is financially linked.

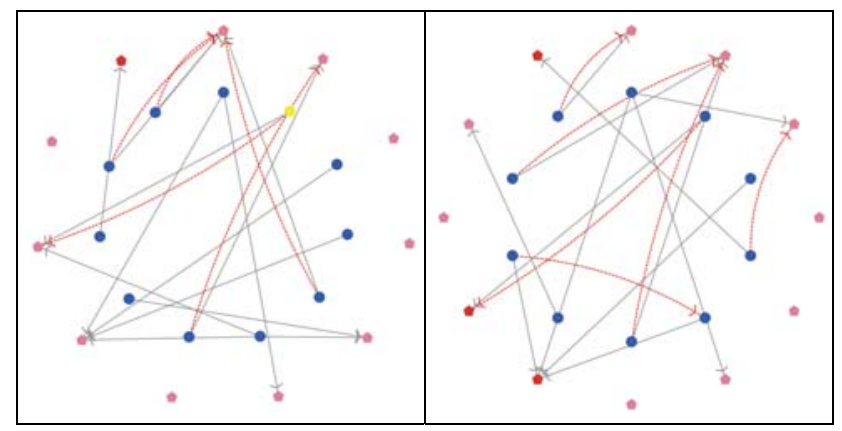

Figure 3. The supply network structures of engine blocks (left: 2002, right: 2012).

Most of the suppliers supply only one assembler, and, as can be seen in the figure, this link is often to assemblers with which they have financial relationships. This corresponds to the argument that in-house manufactured products tend to be those that require high technological knowledge/skills and/or expensive facilities [6]. Assemblers share their core knowledge and skills only with their subsidiaries, and maintain long-term relationships with these companies. The relative nestedness value of the 2012 network was $N_{r}=-1.9$, indicating that the market selection process does not play a part in the emergence of this type of supply network. It appears that other factors (financial relationships and core skills) are more dominant. 
We also found that suppliers in this network tend to have wider product portfolios (i.e. producing many products in various product categories). This suggests that the keiretsu relationships may still be maintained and have significant advantages in the markets of these products.

\section{(c) Products that rely on technological innovation}

Finally, a highly competitive product market was investigated. Figure 4 shows the supply network structures of intake manifolds in 2002 and 2012. This product used to be cast aluminum, but due to advances in technology it is now a resin product. The market has therefore undergone fluctuations. This is reflected by significant changes in the network: the orange-coloured supplier in the 2002 network changed its name, the one in yellow disappeared by a merger, the one in brown disappeared by acquisition, and the one in green in the 2012 network appeared as a result of merger between two companies that decided to enter this market at some point in the period of 2002-2012.

Table 3 shows the number of suppliers, supply links, $N$ and $N_{r}$ of this product market in each of the 5 years. There are more than twice as many suppliers as assemblers, indicating the intense competition. $N$ and $N_{r}$ have been increasing over time, implying that the supply network structure of this product has been changing towards a market with increased sustainability. If this tendency continues, we can expect that some of the suppliers will be eliminated through the market selection process.

In fact, our dataset reveals that all of the companies that went bankrupt in the period of 2002-2012, were producing one, and only one product, categorised as one of these type (c) products. For survival in a severe competitive market, a supplier must widen its product portfolio, or achieve cost or technological advantages of its product over competitors.

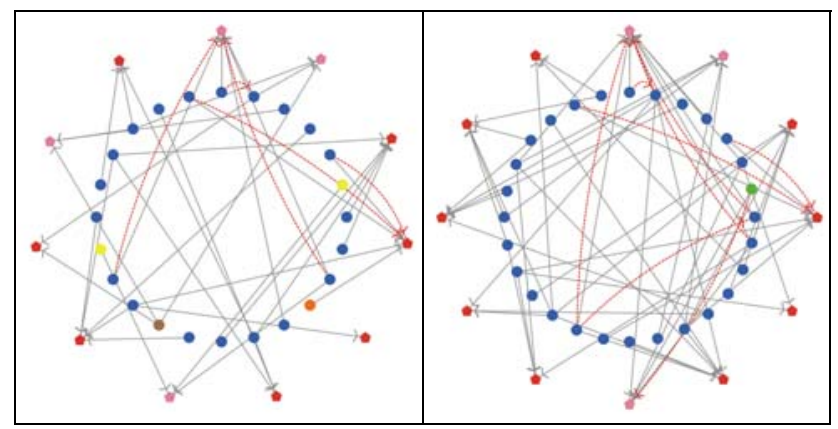

Figure 4. The supply network structures of intake manifolds (left: 2002, right: 2012).

Table 3

Features of the intake manifold supply networks.

\begin{tabular}{|c|c|c|c|c|c|}
\hline & 2002 & 2005 & 2008 & 2010 & 2012 \\
\hline No. of suppliers & 24 & 24 & 26 & 27 & 28 \\
\hline No. of supply links & 37 & 45 & 51 & 57 & 57 \\
\hline$N$ & 15.6 & 21.3 & 25.3 & 23.5 & 21.4 \\
\hline$N_{r}$ & 2.3 & 2.7 & 3.4 & 3.1 & 3.9 \\
\hline
\end{tabular}

\section{Conclusion}

In this paper, we investigated the structural features of automobile parts supply networks and their temporal transitions, based on the complex network perspective. We found that, in the markets of different types of products, supply relationships between assemblers and suppliers are formed differently, reflecting various factors such as product characteristics, technological advancement and financial interdependencies.
The main contribution of this paper is twofold. First, we demonstrated the advantages of analysing empirical data on supply network structures, and applying the complex network approach. The network perspective provides profound insights into the heterogeneity of network structures, and the effects of various factors on the structures, which theory-/simulationbased studies or statistical analysis cannot offer. Second, our detailed time-series data is valuable compared to other data available and analysed to date. The application of the concept of nestedness is also novel, and enabled us to relate the dynamic structural features to market changes.

Finally, as an outlook, we note that using a network perspective to obtain a systematic understanding of supply network structures and their temporal transitions, at both the macro and micro levels, provides many benefits. At the macro level, for example, complex network studies have proposed various metrics to quantify the resilience (a topic of major importance) of a given network, and furthermore resulted in the proposal of methods to make the network more resilient. At the micro level, complex network studies (including this study) have shown that the positioning of an individual company within the network affects its performance and survivability. We believe that future studies of complex supply networks will contribute to companies' strategies for market entrance/exit and production.

\section{Acknowledgements}

This research was partially supported by Service Science, Solutions and Foundation Integrated Research Program, Research Institute of Science and Technology for Society (RISTEX), Science and Technology Agency (JST), Japan.

\section{References}

[1] Ueda, K., Vaario, J., Takeshita, T., Hatono, I., 1999, An emergent synthetic approach to supply network, Ann. CIRP, 48/1:377-380.

[2] Choi, T.Y., Dooley, K.J., Rungtusanatham, M., 2001, Supply networks and complex adaptive systems: control versus emergence. J. Oper. Manag., 19/3: 351-366.

[3] Pathak, S.D., Dilts, D.M., Mahadevan, S., 2009, Investigating population and topological evolution in a complex adaptive supply network, J. Supply Chain Manag., 45/3:54-67.

[4] Mazzola, E., Perrone, G., La Diega, S.N., 2008, Shaping inter-firm collaboration in new product development in the automobile industry: a trade-off between a transaction and relational-based approach, CIRP Ann.-Manuf. Techn., 57/1:485-488.

[5] Cusumano M.A., Takeishi, A., 1991, Supplier relations and management: a survey of Japanese, Japanese-transplant, and U.S. auto plants, Strategic Manage. J. 12:563588.

[6] Fujimoto, T., Takeishi, A., 1994, Jidosha Sangyo 21 Seiki he no Shinario (The Automotive Industry: A Scenario towards the 21st Centry), Tokyo: Seisansei Shuppan.

[7] Fujiki, K., 2001, Kawaru Jidosha Buhin Torihiki: Keiretsu Kaitai (Changing auto parts transactions: the demise of keiretsu), EconomistSha.

[8] Knoke, D., 1990, Political networks: the structural perspective, New York: Cambridge University Press.

[9] Uzzi, B., 1997, Social structure and competition in interfirm networks: the paradox of embeddedness, Administrative Science Quarterly. 42:35-67.

[10] Dyer, J.H., Nobeoka, K., 2000, Creating and managing a high-performance knowledge-sharing network: the Toyota case. Strateg. Manage. J., 21:345 -367.

[11] Lomi, A., Pattison, P., 2006, Manufacturing relations: an empirical study of the organization of production across multiple networks. Organ. Sci. 17/3:313-332.

[12] Kim, Y., Choi, T. Y., Yan, T., Dooley, K., 2011, Structural investigation of supply networks: a social network analysis approach. J. Oper. Manag. 29:194-211.

[13] Kito, T., 2013, Structural robustness of real-world supply chains: a complex network approach, IPSJ TOM, 6/2:174-181.

[14] Jidousha-buhin 200 hinmoku no seisan ryutsu chosa (Survey on production and distribution of 200 auto-parts), IRC (www.eirc.co.jp).

[15] Bascompte,J.,Jordano,P.,Melia'n,C.J., Olesen,J.M., 2003, The nested assembly of plant-animal mutualistic networks, Proc. Natl Acad. Sci. USA, 100:9383-9387.

[16] Almeida-Neto, M., Guimara es,P.,Guimarães, P.R. Jr, Loyola,R.D., Urlich, W.A. 2008, Consistent metric for nestedness analysis in ecological systems: reconciling concept and measurement, Oikos, 117:1227-1239.

[17] Saavedra, S., Strouffer, D.B., Uzzi, B., Bascompte, J., 2011, Strong contributors to network persistence are the most vulnerabile to extinction, Nature, 478:233-235.

[18] Newman, M.E.J., Girvan, M., 2004, Finding and evaluating community structure in networks, Phys. Rev. E, 69/2. 